the old newspaper. He then washes his hands and puts the scissors and suturing materials back in the plastic box.

The RMP takes a syringe from the bag, loads it with what seems to be some medicine, and injects it into the old man's buttocks. He then removes the needle and flushes the syringe by loading it with the water from a container nearby. He then takes another vial from his bag, attaches the needle to it, loads the syringe with the medicine from the vial, and injects it into the other buttock. The syringe is put back into the bag. The RMP informs the family that he will visit them in the evening to inspect the wound. He gives them antibiotics and pain killers and instructs them on their use. He also instructs the family to get a barber and shave around the wound area.

The researcher, who is trained in modern medicine, finds the treatment inappropriate. If he intervenes in this situation, the family's only alternative would be to take the old man to the hospital $9 \mathrm{~km}$ away. Reporting the RMP to the concerned authorities would deprive the tanda of the only source of healthcare available at their doorstep.

\section{Ethical issues}

1. What are the researcher's ethical responsibilities? What are his possible courses of action, and why?

2. The researcher obtained consent from the village representatives to observe the residents of the tanda. Should the researcher have obtained consent from the RMP, who is not from the tanda, to observe his treatment of the old man?

3. The researcher on his next visit in the tanda learns that the old man had died on the fifth day after his fall. Considering the events that the researcher observed, how would the death of the old man reflect on the dilemmas already faced by the researcher?

Acknowledgements: The author thanks Dr Vijayaprasad Gopichandran, Dr Amar Jesani, Dr Rakhi Ghoshal and Dr Ravi Prasad Verma for their comments during the Workshop on Public Health Case Studies held on March 26-27, 2018 at the Achutha Menon Centre for Health Science Studies, SCTIMST, Thiruvananthapuram.

\section{Note}

1 Ethnography is a research tool which describes and interprets the shared and learned patterns of behaviour, beliefs and languages of a group. The aim of ethnography is to provide rich insights and descriptions into the views, beliefs and actions of people through detailed observations and interviews. It involves observations of the group and interviews of participants.

\section{References}

1. Rao $M$, Rao KD, Kumar AS, Chatterjee $M$, Sundararaman T. Human resources for health in India. Lancet. 2011 Feb 12;377(9765):587-98.Doi: 10.1016/S0140-6736(10)61888-0. Epub 2011 Jan 10.

2. George A, lyer A. Unfree markets: Socially embedded informal health providers in northern Karnataka, India. Soc Sci Med. 2013 Nov; 96:297304. doi: 10.1016/j.socscimed.2013.01.022.

3. Pulla P. Are India's quacks the answer to its shortage of doctors? BMJ. 2016 Jan 21;i291. Doi: https://doi.org/10.1136/bmj.i291

4. Nandan D, Agarwal D. Human resources for health in India: Urgent need for reforms. Indian J Community Med. 2012 Nov 15;37(4):205. DOI: 10.4103/0970-0218.103464

5. Roy Burman JJ. Chapter 3, Laman Banjaras: The people. In: Ethnography of a denotified tribe: The Laman Banjaras. New Delhi: Mittal Publications; 2010; pp19-24.

\title{
The non-formal healthcare provider dilemma - to hate the player or the game?
}

\section{RAVI PRASAD VARMA}

Keywords: Informal care provider, harm, professional codes, research objectivity, moral dilemma

The case study by Bevin Vijayan (1) draws attention to a wellrecognised public health problem - trauma and the lack of formal trauma care services in India (2). This is the reality in

Author; Ravi Prasad Varma (rpvarma@sctimst.ac.in), Associate Professor, Sree Chitra Tirunal Institute for Medical Sciences and Technology, Achutha Menon Centre for Health Science Studies, Trivandrum, INDIA.

To cite: Varma RP. The non-formal health care provider dilemma - to hate the player or the game? Indian J Med Ethics. 2020 Jan-Mar;5(1) NS: 17-9. DOI: 10.20529/IJME.2020.013.

Peer Reviewer: An anonymous reviewer

OIndian Journal of Medical Ethics 2020
India for trauma and for many other health problems. Despite decades of dutifully repeating the platitudes of "availability" and "accessibility", the problem is not merely a case of a service being "present" or "absent". A layered milieu of multiple elements related to peoples, systems and cultures determines the services people actually receive. It is here that we see the non-formal healthcare provider, often called a Rural Medical Practitioner (RMP), make an entrance.

Do the practices of these RMPs amount to biomedicine? Biomedically speaking, one would approach the incident referred to in the case study with several concerns. Why did the person fall in the first place? Is it due to an underlying endocrine or metabolic disorder, or perhaps a stroke? What are the possible issues the person has in addition to the scalp laceration - other 
injuries or fractures? What complications are likely - eg intracranial bleeds? Conceivably, aseptic wound repair, infection prevention and control, pain relief, correction of underlying issues and vigilance for complications might have done the person a whole lot of good and he might have been alive today. However, the person ended up getting unsafe injections and sutures which could transmit dreaded diseases like tetanus, Human Immunodeficiency Virus, Hepatitis B or C. The patient and family were led into a sense of reassurance that was possibly false. Money that may have contributed to better care got spent elsewhere. The advice to shave the wound area after the suturing clinches the case for the travesty that took place. As with many biomedical interventions, the jury is still out on whether shaving is really beneficial or not, but it remains a common practice among surgeons in India (3). However, shaving happens before suturing as that is expected to decrease post-operative infection, ease suturing and application of dressings. So, does the "form over function" type of service the man received amount to biomedicine, even in an informal or non-formal sphere? Probably not.

Who can be held accountable for this transgression - the patient or family for not seeking proper care? The non-formal provider / quack? Imagine a river separating a village and a school where children are forced to depend on a substandard country boat to get across. The boat often being overcrowded may capsize and lives may be lost. Who is to be held responsible? The children for overcrowding, or the boatman for offering unsafe transport? Or someone else for the lack of a bridge or a safe ferry? In such situations as that involving the metaphorical boatman or the non-formal care provider, there are probably no heroes to summon or villains to malign. On that account, one should recognise that the formal system that claims proprietorship over biomedical knowledge cannot be absolved of all blame for quackery - the peculiarities of the former partly create the spaces and circumstances for the latter.

Within this frame of reference, the ethical principles that can help guide resolution here are:

\section{Beneficence}

The RMP service may impart satisfaction to the receiver and may be sufficient for wound healing in uncomplicated situations. Potential benefits from the formal system include pain relief, infection control and correction of underlying conditions and complications, and eventually, survival.

\section{Non-maleficence}

The possibilities of harm with the RMP's service, listed earlier, often outweigh the benefits. The formal system has its share of ills too - financial hardships, stigmatising behaviours and medical errors. Providers and systems representing the formal system often put profits over people, either due to a culture of venality or to concerns over the economic sustainability of the system in its current form. The end result is often patients and families being trapped in debt. Some of the potential harms may be almost completely compensated for by the benefits of the intervention. But meanings are not the same for everyone.
Some forms of harm - like those related to physically invasive investigations - may be perceived by health professionals as trivial in relation to the potential benefits. However, these may be perceived as important harm by patients and families.

\section{Paternalism}

The biomedical lens almost axiomatically lends a paternalistic perspective to the issue, as seen in the first half of this commentary. Patients are thus helpless individuals and if they end up placing their trust in the wrong persons, the consequences may be very unfortunate. The power the paradigm holds implies that paternalism also shapes regulations, policies and programmes (4).

\section{Autonomy}

Autonomy is the power of self-determination of the patient and family to make a choice. But does it supplant a paternalistic decision to refer the patient to care which might have saved the patient's life? While patient autonomy increasingly figures in medical discourses, particularly in the West, with calls to at least have a judicious balance of paternalism and autonomy, health systems in India are notoriously paternalistic and patients are passive recipients of care (5).

\section{Dignity}

The acceptability of the practitioner in the tanda suggests that he upholds their dignity. The tribes may have experienced stigmatising behaviours from formal providers, thus influencing their healthcare choices.

\section{Reciprocity}

Money is exchanged for services received - the people are part of the process and this makes the acceptance of service from the RMP more tangible. Treatments in the formal systems are not often patient-centred and shared decision making based on the patient's experiences and expectations is quite rare.

Such situations can be particularly challenging for researchers with a biomedical training, familiar with the Hippocratic injunction "Whatever houses I may visit, I will come for the benefit of the sick" (6). One will need to decide between fidelity to the Oath and fidelity to the protocol. Approaches like ethnography compound the dilemma, as active intervention in such a situation may drastically modify how the community perceives the researcher and thus influence the research process itself. By choosing to be silent, will the silence of the researcher be interpreted as endorsement of the care provided? Will the researcher end up having to impose moral sanctions on himself/ herself if harm occurs subsequently? The ethical requirement of confidentiality and the lack of a clear fiduciary relationship with therapeutic intent between the researcher and the persons involved in this event may absolve the observer from taking remedial action or reporting this event to institutions like State Medical Councils or professional bodies. However, in the event of the researcher being called upon to testify as a witness, he/she may have to be more explicit when legally released from such confidentiality 
requirements (7)

Such dilemmas are likely to be common in public health research, but the accounts may vary based on the researcher's personal values, training and past experiences. Researchers may, therefore, opt for maintaining a diary of such ethical dilemmas they come across in the field and reflect on the choices and possible consequences of their actions. Further, they should initiate discussion with peers and mentors to facilitate better engagement with such issues. If nothing else, they must recognise that while it may be difficult to endorse such practices, the patient and his family members are probably not diminished in front of the non-formal providers the way they might be when they access a formal provider.

\section{References}

1. Vijayan B. Observation of unsafe medical practice during research in a healthcare-deprived area. Indian J Med Ethics. 2020 Jan-Mar; 5(1) NS: 167. DOI: 10.20529/IJME.2020.012
2. Roy N, Gerdin M, Ghosh S, Gupta A, Kumar V, Khajanchi M, Schneider EB, Gruen R, Tomson G, von Schreeb J. 30-day in-hospital trauma mortality in four urban university hospitals using an Indian trauma registry. World J Surg. 2016 Jun;40(6):1299-307. Doi: 10.1007/s00268-016-3452-y.

3. Sebastian S. Does preoperative scalp shaving result in fewer postoperative wound infections when compared with no scalp shaving? A systematic review. J Neurosci Nurs. 2012 Jun;44(3):149-56. Doi: 10.1097/JNN.0b013e31825106d2.

4. Aasen EM, Dahl BM. Construction of patients' position in Norway's Patients' Rights Act. Nurs Ethics. 2019 Nov-Dec; 26(7-8):2278-2287. doi: 10.1177/0969733018791345. Epub 2018 Aug 23.

5. Fochsen G, Deshpande K, Thorson A.Power imbalance and consumerism in the doctor-patient relationship: health care providers' experiences of patient encounters in a rural district in India. Qual Health Res. 2006 Nov;16(9):1236-51.

6. Markel H."I swear by Apollo"—on taking the Hippocratic Oath. N Engl J Med. 2004 May 13;350(20):2026-9.

7. Surmiak A. Should we maintain or break confidentiality? The choices made by social researchers in the context of law violation and harm. $J$ Acad Ethics. 2019 Jul:1-9. DOI: 10.1007/s10805-019-09336-2

\section{Use of critical reflection as a research method: A case of research-induced distress?}

\section{MALU MOHAN}

Keywords: Critical reflection, qualitative research, diary entries, professional distress, research-induced distress

\section{Introduction and context}

Critical reflection is widely used in qualitative research (1) It helps us understand participants' internal dialogues and analyse their thought processes. The use of oral or written reflections is a well-documented data collection method, particularly in educational research (2).

This case study presents the ethical distress of a public health researcher engaged in a study that sought to explore the preparedness of health professionals who had recently graduated from a particular branch of health services, to practise independently.

\section{The case}

The drastic commercialisation in this branch of medical education over the past three decades has resulted in a

Author: Malu Mohan (dr.malumohan@gmail.com), Research Coordinator Project on Decentralisation and Health: Lessons from the Kerala Experience, Kerala Institute of Local Administration, Thrissur, Kerala, INDIA.

To cite: Mohan M. Use of critical reflection as a research method - a case of research-induced distress? Indian J Med Ethics. 2020 Jan-Mar; 5(1) NS:19-20. DOI: 10.20529/IJME.2020.014.

OIndian Journal of Medical Ethics 2020. tremendous increase in the number of graduates and of private practices, and intensified competition in the private job market. This called for an enquiry into how prepared graduates were for these changes, and to provide quality care.

Recent graduates with independent practices were asked to record their daily clinical practice experiences for eight weeks in diary entries. They were asked to introspect on how their strengths and weaknesses as practitioners could have contributed to these experiences. They were also asked to identify those professional areas in which they considered themselves inadequately prepared for independent practice. Diary entries were to be reviewed at two weeks, with a second review along with face-to-face interviews at six weeks, and a third review at eight weeks. Apart from the time and effort that the participants had to invest, no other potential harm was anticipated from the study.

On preliminary review at two weeks, the researcher found that the diary entries were superficial, with hardly any reflection. She then impressed upon the participants the need for honest introspection and detailed recording.

After another four weeks, the researcher collected the diaries for analysis and found that the entries had become more detailed. In addition to reflections about daily clinical happenings, they included confessions of participants' insecurities as professionals responsible for the lives of other human beings, particularly because of the quality of their 